\title{
Nuove tecniche di transgenesi e imaging: neuro e nefro-applicazioni. Parte 2
}

\author{
Tommaso Alterini ${ }^{1}$, Duccio Lombardi ${ }^{2}$ \\ ${ }^{1}$ Dottorando in Ingegneria Ottica, Centre for Sensors, Instruments and Systems Development, Universitat Politecnica de Catalunya, Barcel- \\ lonatech, Barcellona - Spagna \\ ${ }^{2}$ Assegnista di Ricerca post-Dottorato, Dipartimento di Scienze Biomediche, Sperimentali e Cliniche, Unità di Nefrologia, Università degli Studi \\ di Firenze, Firenze
}

\begin{abstract}
New techniques of transgenesis and imaging: applications in Neurology and Nephrology. Part II
Strengthened by the analysis of genetic engineering approaches and strategies related to transgenic animal models and of the most cutting-edge imaging techniques, this article will be centered on the impact that such applications have and will have in research and, as a consequence, in the clinical area.

In order to show how identical techniques can be applied in different contexts for the study of different organs and tissues, in this issue the applications of these technologies in the anatomical and functional studies of distinct organs such as the kidney and the central nervous system will be analyzed. In particular, concrete examples about the kidney and regenerative nephrology will be exploited to show how different techniques can lead to similar, or diametrically opposite data, depending on the approach used to reach the specific research or diagnostic goal.
\end{abstract}

Keywords: Clinical applications, Cutting-edge imaging techniques, Kidney, Transgenic strategies

\section{Introduzione}

Nella prima parte di questo articolo (1) sono stati introdotti tutti i concetti, le possibilità e le strategie che possono essere usati nel campo della ricerca grazie all'impiego di modelli animali transgenici e di tecniche avanzate di microscopia. Forti delle basi teoriche su cui si basano i sistemi transgenici e quelli di imaging, in questo contributo saranno analizzate le possibili applicazioni nel mondo della ricerca, così come le ricadute che queste hanno in campo clinico, con particolare riferimento all'area nefrologica.

Vale la pena ricordare che, quando si parla di animale transgenico, si fa riferimento a un animale in cui sono stati modificati, rimossi o aggiunti uno o più determinati elementi genetici esogeni al fine di studiare come la delezione, l'iperespressione o le modifiche a carico di un dato gene affliggano il fenotipo dell'animale, determinando, per esempio, speci-

\section{Accepted: April 22, 2016}

Published online: May 12, 2016

Indirizzo per la corrispondenza:

Dr. Tommaso Alterini

Universitat Politecnica de Catalunya

Barcelonatech

Rambla Sant Nebridi 10

08222 Terrassa, Barcelona, Spain

t.alterini@gmail.com fiche patologie $(1,2)$. Analogamente, è possibile inserire nel genoma animale un cosiddetto gene reporter che codifica per molecole tracciabili, le quali consentono di individuare specificamente determinate popolazioni cellulari e di studiarne il comportamento in situazioni fisiologiche o patologiche.

Data la versatilità dell'utilizzo della transgenesi e le grandi specificità e accuratezza legate all'uso di sistemi che sfruttano modifiche genetiche, è facile intuire le vaste potenzialità che derivano dal suo impiego per lo studio di sistemi biologici complessi come interi organi od organismi. Tuttavia, al fine di sfruttare al meglio tutti i possibili vantaggi derivanti dall'ingegnerizzazione genetica, è necessario che questa lavori in stretta sinergia con tecniche di imaging in continuo sviluppo, così da poter fornire strumentazioni sempre più veloci, precise e affidabili.

Oggi, le tecniche di imaging riescono a spaziare in svariati ordini di grandezza, sia temporalmente che spazialmente, riuscendo a ottenere in poco tempo tomografie di intere sezioni anatomiche a "bassa" risoluzione (risonanza magnetica) (3) o ricostruzioni di piccole porzioni di tessuto, apprezzando dettagli dell'ordine dei nanometri (microscopia elettronica) (4) (Fig. 1). Tra questi due estremi ci sono tutte quelle tecniche di microscopia ottica a scansione, come la microscopia confocale, a multifotoni o a foglio di luce, che permettono di avere immagini con risoluzioni cellulari (nell'ordine dei micrometri) e un ottimo contrasto, riducendo, allo stesso tempo, la necessità di sezionamento artificiale che introduce arte- 


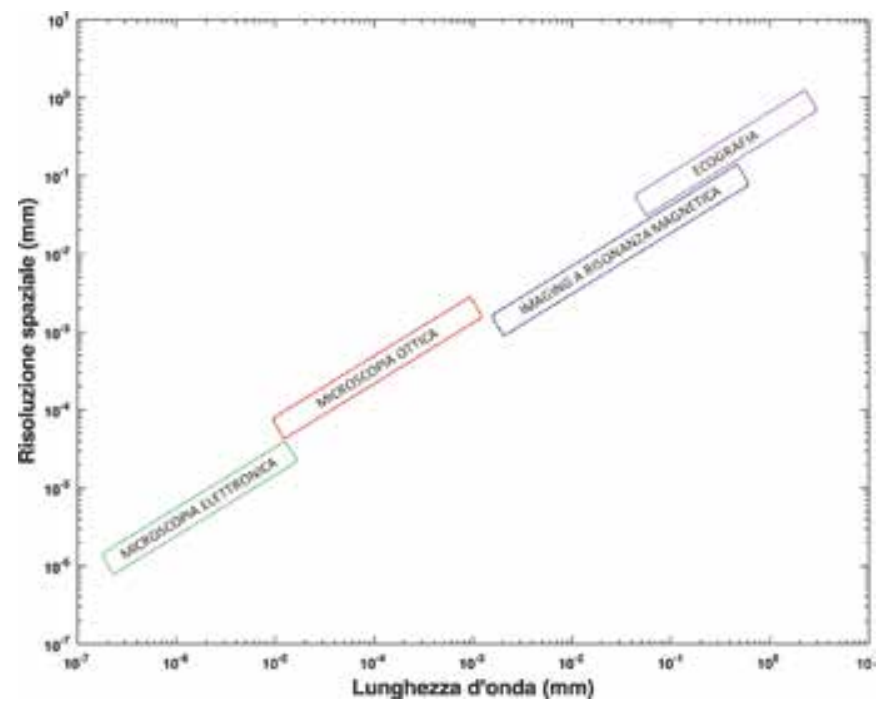

Fig. 1 - Rappresentazione schematica del legame fra lunghezza d'onda impiegata e risoluzione spaziale delle principali tecniche di imaging. Dal grafico è possibile ricavare, a grandi linee, che la lunghezza d'onda impiegata dalla specifica tecnica di imaging è legata al massimo potere risolutivo ottenibile con quella tecnica.

fatti e limita l'affidabilità della ricostruzione su campioni spessi (5). È pertanto facile intuire quanto sia determinante scegliere con accuratezza la tecnica di imaging più adatta allo scopo prefissato. Ciò è dovuto al fatto che esistono limiti fisici imprescindibili dovuti all'essenza ultima della radiazione e della materia, i quali obbligano a cercare il "giusto" compromesso tra velocità di scansione, alte risoluzioni, capacità di penetrazione di un tessuto e invasività dell'analisi (6).

Per esempio, tecniche come la risonanza magnetica nucleare (MRI, Magnetic Resonance Imaging) sono poco invasive, in quanto, per ottenere immagini tridimensionali di interi organi o ampi tessuti, sfruttano campi magnetici di eccitazione a radiofrequenza (lunghezze d'onda di alcuni $\mathrm{mm}$ ). Per questa stessa ragione, tali tecniche non sono in grado di garantire risoluzioni inferiori ad alcune centinaia di micrometri $(\mu \mathrm{m})$, ma permettono, tuttavia, di effettuare imaging su organismi viventi raggiungendo profondità impensabili per la radiazione visibile sfruttata dalla microscopia ottica (7). Quest'ultima, infatti, sfruttando lunghezze d'onda $(\lambda)$ di eccitazione dell'ordine di alcune centinaia di nanometri $(\mathrm{nm})$, garantisce un maggiore potere risolutivo ma, allo stesso tempo, interagisce con tutte le molecole che incontra e, pertanto, non riesce ad arrivare a bersagli troppo in profondità. Questo limita l'utilizzo della microscopia ottica, fra cui la microscopia a fluorescenza, a sezioni di spessore relativamente ridotto, ma, allo stesso tempo, consente di studiare processi biologici microscopici con estrema specificità e precisione.

Scopo di questo contributo è quello di presentare, tramite vari esempi, come le tecniche di transgenesi, quando associate alle più recenti innovazioni nel campo dell'imaging, possano essere impiegate e sfruttate al meglio per rispondere a quesiti di natura biologica, così da analizzare nei più fini dettagli processi che coinvolgono da interi tessuti al singolo componente di una sola cellula. Soprattutto, sarà evidenziato che, mentre le innovazioni legate ad animali geneticamente modificati trovano la loro principale applicazione nel mondo della ricerca, le ricadute delle scoperte fatte impiegando l'associazione di transgenesi e imaging sono il preludio all'applicazione in ambito clinico di strumentazioni sempre più avanzate $e$ che consentono indagini e diagnosi ogni giorno più accurate e affidabili (8).

\section{Ricerca pre-clinica, imaging a fluorescenza e rene: stu- dio della dinamicità renale}

Attualmente, transgenesi e microscopia a fluorescenza stanno avendo un grandissimo successo nel campo della ricerca, specialmente per quanto riguarda la ricostruzione e l'analisi di tessuti sia ex-vivo che in vivo, ove si riescono a raggiungere profondità maggiori di un millimetro senza la necessità di un sezionamento artificiale del campione e di sacrificio dell'animale (9). Nella microscopia a fluorescenza in vivo, infatti, i processi cellulari sono osservati direttamente nel momento in cui avvengono e in un contesto quasi del tutto fisiologico.

Il grande vantaggio delle tecniche di microscopia a fluorescenza è che sono corroborate da un largo impiego ormai da più di mezzo secolo, e dal fatto che, nell'ultima decade, hanno visto un poderoso sviluppo ed avanzamento tecnico. Inoltre, la strumentazione necessaria è facilmente reperibile sul mercato e si basa su un tool estremamente versatile: la fluorescenza (7).

Un ottimo esempio di come possa essere sfrutta la fluorescenza ci è data dalla microscopia confocale. Grazie alla microscopia confocale è infatti possibile visualizzare, per esempio, variazioni ultrastrutturali nella morfologia podocitaria, come quelle associate alla glomerulopatia a lesioni minime. $\mathrm{Se}$, infatti, fino a pochi anni fa, l'appiattimento cellulare e la ritrazione dei processi pedicellari tipicamente associati a tale patologia sono stati apprezzabili solo grazie alla microscopia elettronica, adesso la scoperta di fluorofori sempre a maggior intensità di emissione e lo sviluppo di nuove macchine per l'imaging fanno sì che tale assioma non sia più vero (10). Va, tuttavia, ricordato che la microscopia elettronica rimane la migliore tecnica di microscopia in termini di risoluzione; malgrado ciò, essa presenta tempi di scansione che la rendono proibitiva per analisi su larga scala, permette l'analisi solo di piccole porzioni di tessuto e impedisce ricostruzioni tridimensionali fedeli a causa dei difetti artificiali dovuti al sezionamento necessario al processamento del tessuto (4). A causa del fatto che, fino a poco tempo fa, le conoscenze riguardanti strutture e processi biologici di scala cellulare e subcellulare erano completamente basate su tale tecnica poco routinaria, poco era noto di quanto avvenisse 

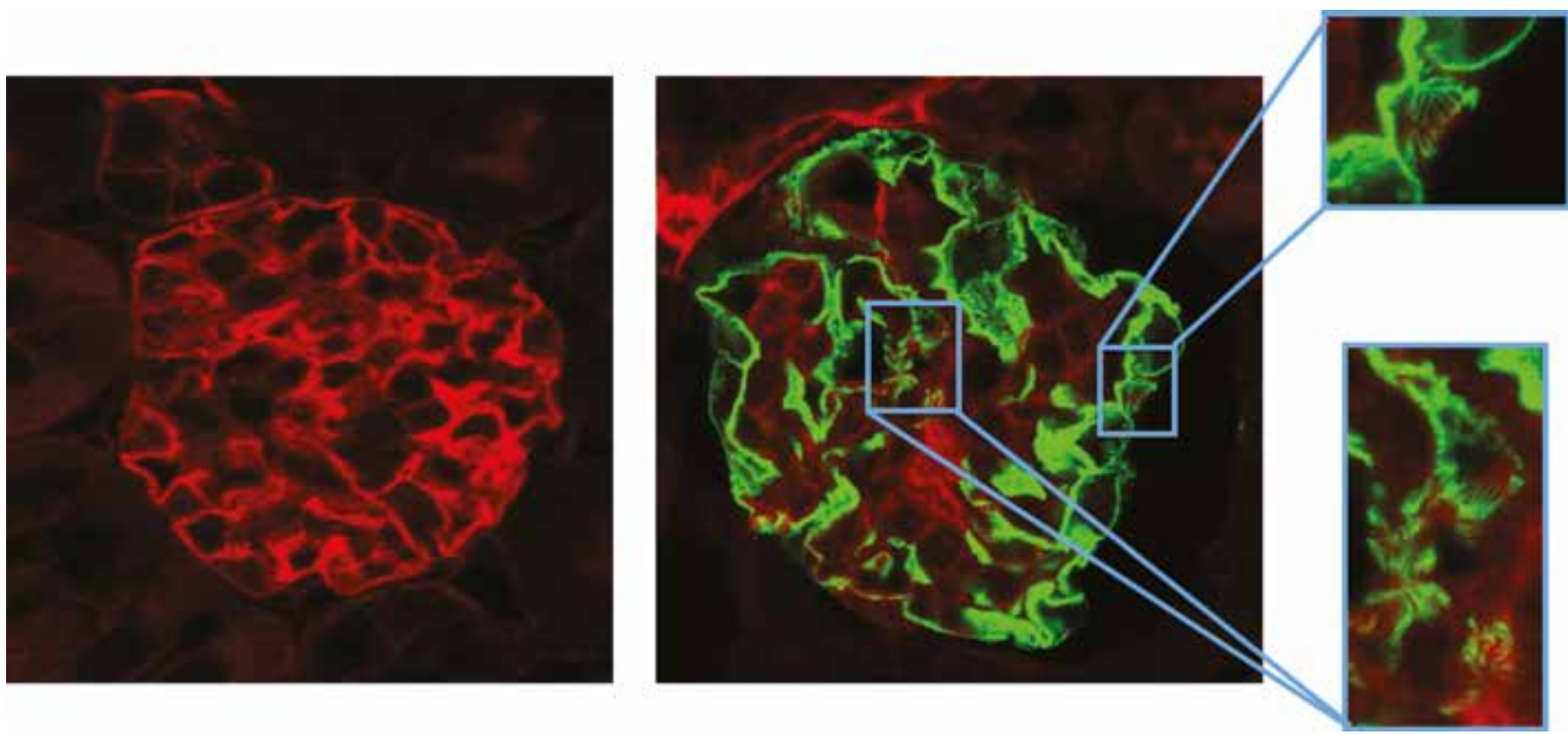

Fig. 2 - Il sistema $\mathrm{mT} / \mathrm{mG}$ e la visualizzazione podocitaria: posto sotto il promotore della podocina, questo transgene permette, in seguito ad induzione nei modelli condizionali, o in seguito a differenziazione in senso podocitario nei sistemi costitutivi, di visualizzare i soli podociti in verde. Il forte segnale di fluorescenza del verde, come visualizzabile dai pannelli sulla destra, permette di arrivare a visualizzare dettagli senza precedenti, quali i processi pedicellari del podocita stesso. Modificato da Lombardi D, Lasagni L. "Transgenic Strategies to Study Podocyte Loss and Regeneration". Stem Cells International vol. 2015, Article ID 678347, 2015. doi: 10.1155/2015/678347.

a livello ultrastrutturale su interi organi: o era prediletta un'analisi più macroscopica o, focalizzandosi a livello intracellulare, era persa la visione d'insieme. Nelle ultime due decadi, tuttavia, le tecniche di microscopia a fluorescenza sono entrate prepotentemente in scena, sia grazie allo sviluppo di fluorofori ad alte intensità di emissione e resistenza al fotodanneggiamento che grazie allo sviluppo di tecnologie di imaging sempre meno invasive e più veloci, come la microscopia a multifotoni (MPM, Multiphoton Microscopy), la microscopia confocale o la microscopia a foglio di luce (9). Tutte queste tecniche permettono, infatti, di effettuare analisi altrimenti impossibili con la microscopia elettronica, anche se con un inferiore potere risolutivo dovuto all'effetto della diffrazione. Malgrado ciò, anche il limite della diffrazione intrinsecamente legato alla luce può essere parzialmente aggirato utilizzando tecniche ottiche a super-risoluzione come la STED (Stimulated Emission Depletion) o la PALM (PhotoActivated Localization Microscopy) $(11,12)$, le quali sfruttano alte intensità di luce rispetto alle tecniche di microscopia a fluorescenza convenzionali e riescono ad acquisire immagini con risoluzioni non troppo distanti da quelle della microscopia elettronica (decine di $\mathrm{nm}$ ) (13).

Un esempio concreto da cui partire per analizzare come le nuove soluzioni legate alla transgenesi e all'imaging stiano rivoluzionando la ricerca biomedica è il sistema $\mathrm{mT} / \mathrm{mG}$ ( $\mathrm{mem}$ braneTomato/membraneGreen), già introdotto nella prima parte di questo articolo (1). Tale transgene permette, infatti, di individuare specifici pool cellulari di interesse differenziandoli e contrastandoli rispetto a tutte le restanti cellule dell'organismo dell'animale geneticamente ingegnerizzato. Infatti, tutte le cellule dell'animale esprimono costitutivamente sin dalla nascita il fluoroforo rosso TomatoRed, ma, qualora acquisiscano per differenziazione cellulare l'espressione del promotore che guida l'intero sistema transgenico o nel caso siano positive al promotore in questione e siano indotte mediante l'uso di specifiche molecole, le cellule d'interesse passano dall'espressione del rosso a quella del verde (GFP, Green Fluorescent Protein) $(1,2)$. Ciò è dovuto a una ricombinazione Cre-mediata che porta, in ultima analisi, ad avere un netto contrasto in grado di incrementare fortemente la capacità di visualizzazione dei più fini dettagli cellulari e che garantisce, inoltre, di individuare specificamente il solo pool cellulare d'interesse grazie all'impiego di promotori cellula-specifici capaci di attivare tutto il sistema ricombinativo $(1,2)$. Infatti, come visibile nella Figura 2, in cui il promotore impiegato è il gene della podocina, le sole cellule a essere marcate in verde sono i podociti, dei quali, grazie all'elevata intensità di fluorescenza data dalla forma enhanced della GFP (eGFP), possono essere visualizzati dettagli tanto fini quali i processi pedicellari primari (Fig. 2, dettagli in verde). II principale vantaggio legato a tale sistema transgenico è che, come già accennato, basandosi sulla specifica espressione di fluorofori, rende possibile l'analisi tissutale mediante tecniche di microscopia a fluorescenza come la microscopia confocale. Tale tipo di microscopia permette di 


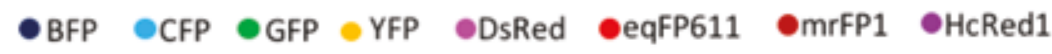
SPETTRI DI ASSORBIMENTO

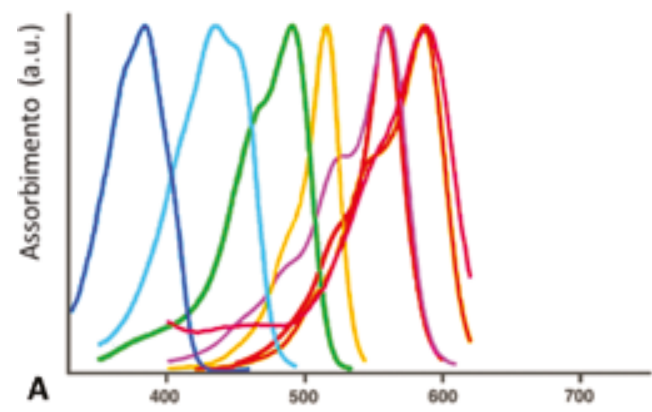

\section{SPETTRI DI EMISSIONE}

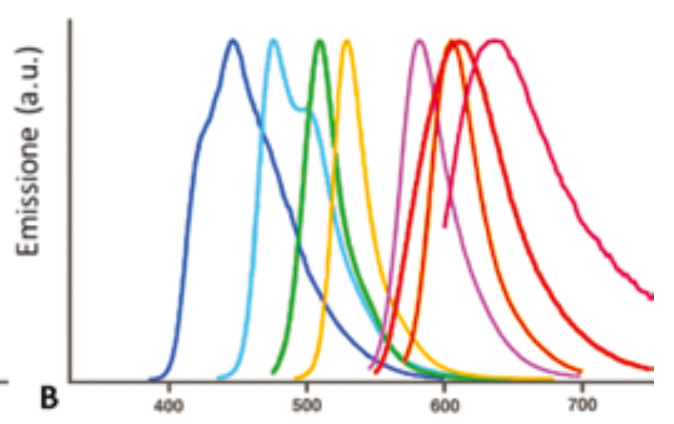

Fig. 3 - Rappresentazione di alcuni spettri di assorbimento (A) e di emissione (B) dei principali fluorofori usati in ricerca biomedica. Caratteristica tipica della fluorescenza è che, coinvolgendo stati energetici vibrazionali non radiativi che fanno perdere energia al fluoroforo eccitato, gli spettri di assorbimento in eccitazione hanno $\lambda$ minori rispetto a quelli in emissione, come visibile confrontando i due grafici. Questo permette di rilevare l'emissione fluorescente su una $\lambda$ differente da quella con cui il fluoroforo è stato eccitato. ottenere alte risoluzioni spaziali e un'alta qualità delle immagini, in quanto è in grado di combinare un sistema di scansione di un fascio laser focalizzato (in eccitazione) con un sistema di sezionamento ottico (in rivelazione) capace di eliminare la maggior parte della luce fuori fuoco o diffusa $(1,14)$. Infatti, come visibile nella Figura 2, l'immagine non presenta zone fuori fuoco e lo sfondo è ben contrastato rispetto alla zona marcata. Sempre grazie al sezionamento ottico, con la microscopia confocale, raccogliendo la fluorescenza emessa con lo stesso obiettivo usato per l'eccitazione, è possibile ottenere fini ricostruzioni 3D di tutto il campione fino a profondità di centinaia di micrometri. Ciò è effettuato grazie alla sovrapposizione sequenziale di tutti i piani che sono progressivamente scansionati muovendo il campione lungo l'asse di rivelazione (processo di z-stack).

La microscopia confocale è, senza dubbio, la tecnica maggiormente impiegata e commercialmente più diffusa tra tutte le tecniche di imaging che sfruttano la fluorescenza al fine di ottenere immagini con elevata risoluzione. Tuttavia, qualora si intenda creare ricostruzioni tridimensionali del campione e siano, quindi, eseguite svariate scansioni su differenti piani (z-stack) all'interno del campione, lo stesso punto è illuminato più e più volte, evento capace sia di indurre fotodanneggiamento (perdita di intensità della fluorescenza) che di incrementare la quantità di luce diffusa, con conseguente peggioramento della qualità dell'immagine (15).

Una tecnica di microscopia, già esaminata nel precedente contributo, in grado di ridurre notevolmente il fotodanneggiamento e di penetrare con maggiore profondità nel tessuto in analisi è la microscopia a multifotoni $(1,16)$. A differenza della microscopia confocale, la MPM limita il fotodanneggiamento, in quanto sfrutta l'assorbimento di due o più fotoni per eccitare una molecola fluorescente: ciò avviene solo in zone spazialmente molto ristrette del campione, dove la densità di fotoni è estremamente elevata.

La tecnica di microscopia a multifotoni più utilizzata è senza dubbio quella a due fotoni, chiamata così perché eccita $\mathrm{i}$ fluorofori del campione mediante l'assorbimento di due fotoni nello stesso istante di tempo (17). Per ottenere la densità di radiazione di eccitazione necessaria per l'assorbimento da parte del solito fluoroforo di due fotoni allo stesso tempo, non è sufficiente confinare spazialmente un fascio laser grazie alla sua focalizzazione sul preparato, ma è necessario anche l'utilizzo di sorgenti laser impulsate che sono in grado di limitare l'esposizione ad un gran numero di fotoni nel tempo. Tuttavia, I'utilizzo di queste sorgenti laser, purtroppo, ha dei costi elevatissimi nonostante si tratti di una tecnica commercialmente disponibile da molto tempo e, per questo, molto spesso si tende a preferire la microscopia confocale rispetto a quella a due fotoni. Come già visto in precedenza, ogni tecnica di microscopia presenta vantaggi e relativi svantaggi: la microscopia confocale, per esempio, non può essere impiegata quando è necessario effettuare analisi del tessuto in vivo in profondità o qualora sia imperativo limitare il fotodanneggiamento. In questi casi, la microscopia a due fotoni, essendo basata sul principio dell'assorbimento di due quanti di luce, permette la conservazione dell'energia e riduce fortemente la perdita di intensità di fluorescenza del fluoroforo, in quanto utilizza laser a $\lambda$ doppie (energia per fotone dimezzata) rispetto alla microscopia a singolo fotone (o confocale). Inoltre, impiegando lunghezze d'onda maggiori, oltre ad aumentare il contrasto poiché eccita una ristretta zona del campione, la microscopia a due fotoni porta a notevoli incrementi nelle profondità di tessuto raggiungibili, mantenendo però, allo stesso tempo, il potere risolutivo di un microscopio confocale. Purtroppo, anche con questa tecnica sono mantenute le limitazioni temporali dovute alla necessità di scansionamento punto per punto del tessuto che si intende studiare: ciò può, tuttavia, essere aggirato grazie a tecniche a campo largo, come la microscopia a foglio di luce, le quali permettono di acquisire interi campi ogni volta che il campione è esposto alla luce di eccitazione $(1,18)$.

Un'altra caratteristica da non trascurare di tutte le tecniche di microscopia basate sulla fluorescenza è il vantaggio di eccitare fluorofori con radiazioni di lunghezze d'onda spettralmente molto lontane l'una dall'altra. Infatti, nella microscopia a fluorescenza, potendo disporre di differenti fluorofori che assorbono ed emettono a $\lambda$ diverse (Fig. 3) e impiegando filtri 

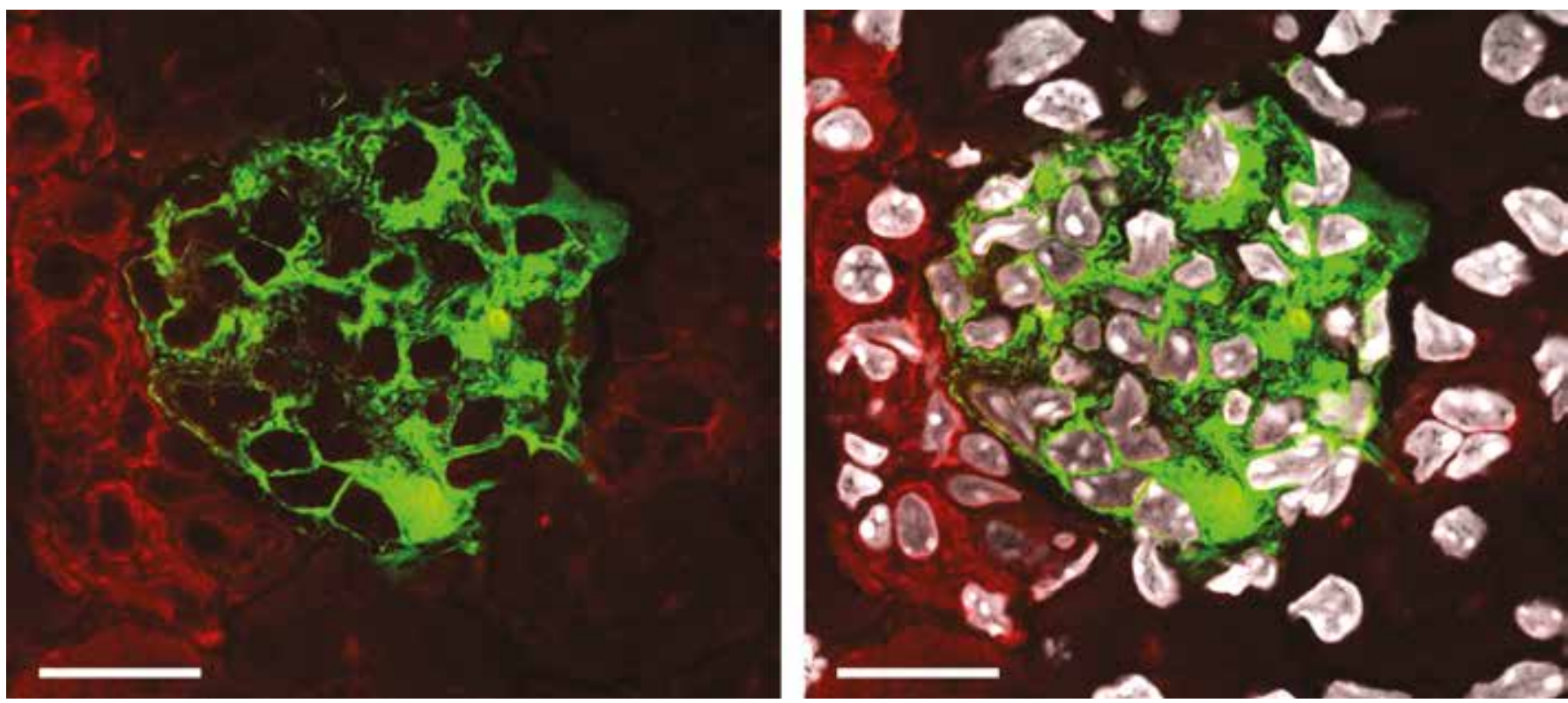

Fig. 4 - Microscopia confocale e microscopia a due fotoni: sistema reporter $\mathrm{mT} / \mathrm{mG}$ posto sotto il controllo del gene codificante la podocina e analizzato mediante microscopia confocale (sinistra) e a due fotoni (destra). Il grande vantaggio della microscopia a due fotoni è che, impiegando un laser impulsato, si rende non nulla la probabilità di avere assorbimenti contemporanei di due fotoni da parte dello stesso fluoroforo: è, quindi, possibile eccitare molecole nel lontano ultravioletto con un laser nel vicino infrarosso. In questo caso, il colorante nucleare DAPI, assorbente a $365 \mathrm{~nm}$, viene eccitato, senza che interferisca con i segnali in fluorescenza degli altri fluorofori, con un laser impulsato intorno a $700 \mathrm{~nm}$ e, quindi, lontano da tutte le transizioni a singolo fotone connesse al sistema $\mathrm{mT} / \mathrm{mG}$. È, così, possibile differenziare nettamente i fluorofori, aumentando anche la larghezza dello spettro a cui si può fare riferimento. Scale bar 20 um.

ottici in rivelazione che permettono di raccogliere solo la luce di fluorescenza di interesse, i segnali provenienti da differenti molecole fluorescenti, come nel sistema $m T / m G$, sono letti singolarmente e non appaiono sovrapposti. Ciò permette di associare i differenti segnali in fluorescenza a diverse popolazioni cellulari o, addirittura, a diverse strutture di una stessa cellula, garantendo, così, un'elevata precisione e un'estrema affidabilità nel riconoscimento delle componenti che vogliono essere studiate indipendentemente (7).

Un ottimo esempio di come segnali differenti siano letti indipendentemente, garantendo, così, alta specificità e grande capacità risolutiva, è visibile nella Figura 4 , in cui sono presenti due immagini acquisite, sullo stesso glomerulo in un modello murino transgenico per $\mathrm{mT} / \mathrm{mG}$, ma con due tecniche diverse. Sulla sinistra, l'acquisizione è stata realizzata in microscopia confocale illuminando il campione con un laser a $488 \mathrm{~nm}$ per leggere in verde il segnale associato ai podociti, e con un laser a $543 \mathrm{~nm}$ che, emettendo nel rosso, permette di individuare tutto ciò che non è podocita. Al contrario, sulla destra, il glomerulo in questione è stato acquisito mediante una microscopia a due fotoni, la quale sfrutta una lunghezza d'onda d'eccitazione di $700 \mathrm{~nm}$ (vicino infrarosso), eccitando i fluorofori che assorbono nel vicino ultravioletto $(358 \mathrm{~nm})$ grazie all'assorbimento contemporaneo di due fotoni $(358 \times 2$ $=716 \mathrm{~nm}$ ). L'impiego di tale lunghezza d'onda, molto lontana spettralmente rispetto alle lunghezza d'onda di rosso e verde, permette di marcare i nuclei cellulari con il colorante nucleare DAPI (4',6-diamidino-2-phenylindole) e di visualizzare anche tale struttura subcellulare tramite un segnale distinto e non sovrapposto alle componenti spettrali di altri fluorofori (7).

Proprio grazie all'impiego di questo modello transgenico e all'uso della MPM, Hackl et al. (19), hanno dimostrato che il nefrone non è un'unità funzionale "statica" e con capacità rigenerative limitate o totalmente assenti, contrariamente a quanto generalmente assunto. Infatti, nel lavoro, gli Autori sono stati in grado di evidenziare che, in un modello murino Podocina- $m T / m G$ con danno da ostruzione ureterale (UUO, Unilateral Ureteral Obstruction), conseguentemente al danno, parte dei podociti si sposta all'interno del tuft glomerulare formando cluster multicellulari (19). La nota più interessante di tale osservazione è che tale dinamismo cellulare è stato osservato nello stesso glomerulo a vari tempi sperimentali: è stato, in questo modo, possibile valutare le variazioni che si hanno sempre sulla stessa struttura anatomica, riducendo la variabilità che si può avere tra glomerulo e glomerulo nella risposta al danno. Per fare ciò, gli autori hanno esteriorizzato il rene dell'animale anestetizzato, posto l'organo sotto il microscopio e, dopo aver scelto l'area da cui prendere le immagini, aumentato la potenza del fascio laser focalizzandolo su un punto facilmente riconoscibile (per esempio un glomerulo adiacente), il quale, una volta "bruciato", ha lasciato uno spot cicatriziale fluorescente e indelebile nel tessuto. In questo 
modo, è stato possibile ritrovare facilmente il corretto posizionamento per osservare lo stesso glomerulo nei successivi time point.

Inoltre, Hackl et al., sempre sfruttando la MPM sullo stesso modello in vivo con fluorofori a differenti spettri di emissione, hanno potuto tracciare i podociti a tempi differenti, dimostrando come, a 1-2 settimane dall'induzione del danno, i podociti stessi si distacchino dalla membrana basale glomerulare, imbocchino i primi tratti del tubulo prossimale (19), si dirigano verso i dotti collettori e, infine, siano persi nelle urine, come già osservato in precedenza in altri lavori $(20,21)$. Ciò che, però, ha maggiormente sorpreso di questo lavoro è che gli Autori hanno scoperto un fenomeno biologico mai descritto prima, in un differente modello murino in cui, però, a essere marcate in verde sono le cellule che compongono l'epitelio della capsula di Bowman (PEC, Parietal Epithelial Cells), già descritte come progenitori di natura staminale sia nell'uomo che nel topo $(22,23)$. Infatti, è stato osservato che, da tali PEC marcate in verde grazie all'uso del promotore PEPCK (Phosphoenolpyruvate carboxykinase) $(19,24)$, in seguito ad induzione di UUO si formavano delle connessioni nanotubulari che attraversavano l'intero spazio di Bowman fino a entrare a contatto con i sottostanti podociti. Tali connessioni, con diametro quasi inferiore al limite di risoluzione di $350 \mathrm{~nm}$ e di lunghezza anche maggiore di $25 \mu \mathrm{m}$, potrebbero servire a controllare lo stato di salute podocitaria o al transito intercellulare di molecole e organelli, così come sostenuto dagli stessi Autori (19), sarebbero ossia sensori di danno e/o meccanismi tramite i quali cellule sane passerebbero componenti cellulari volte alla sopravvivenza delle cellule danneggiate. Inoltre, è plausibile che tali connessioni siano i primi contatti atti a formare ponti cellulari al fine di sostituire in maniera estremamente rapida i podociti persi (25). Tali strutture sono di difficile rivelazione a causa delle loro dimensioni estremamente ridotte e, spesso, con tecniche di imaging a fluorescenza possono non essere individuate durante l'analisi o non illuminate con abbastanza potenza, motivo per cui la ricerca è sempre più orientata verso tecniche e tecnologie ad alta intensità di fluorescenza e che limitano il più possibile il fotodanneggiamento.

Oltre che per le analisi di natura qualitativa, come quella svolta da Hackl et al., la tecnologia MPM può essere impiegata anche per analisi di natura quantitativa, sia in condizioni fisiologiche che in condizioni patologiche $(26,27)$. Di particolare interesse sono le valutazioni delle oscillazioni temporali nel tasso di filtrazione di un singolo nefrone, le variazioni del flusso ematico e tubulare, i cambiamenti nelle resistenze vascolari e nella permeabilità capillare e lo studio delle dinamiche del $\mathrm{Calcio}^{2+}\left(\mathrm{Ca}^{2+}\right)$ (27-30). Queste analisi permettono di vedere come determinate condizioni o patologie influiscano sulla GFR (Glomerular Filtration Rate), sull'incremento/ decremento del flusso di pre-urina intratubulare, e come alcuni farmaci modifichino le resistenze vascolari glomerulari o abbiano effetti sulla permeabilità della barriera di filtrazio- ne glomerulare (o GFB, Glomerular Filtration Barrier) $(31,32)$.

Per esempio, sebbene le analisi della concentrazioni e delle dinamiche del $\mathrm{Ca}^{2+}$ siano ancora in una fase embrionale per quanto riguarda il rene, è stato recentemente dimostrato che, mentre la concentrazione di $\mathrm{Ca}^{2+}$ è molto bassa nei podociti in condizioni fisiologiche, questa aumenta notevolmente nei podociti adiacenti a quello danneggiato in seguito all'induzione di danno podocitario (30). Infatti, Burford et al., sfruttando un modello transgenico in cui il promotore della podocina porta all'espressione di una forma modificata della calmodulina che fluoresce quando legata al $\mathrm{Ca}^{2+}$ (GCaMP3) hanno dimostrato che il danno podocitario promuove un fenomeno a cascata generante un'onda di $\mathrm{Ca}^{2+}$, che viene trasmessa sequenzialmente da un podocita a quello adiacente, causando un incremento nella concentrazione di $\mathrm{Ca}^{2+}$ intracellulare (30). Tale influsso di $\mathrm{Ca}^{2+}$ è, poi, responsabile della contrazione del tuft glomerulare al quale segue una maggiore permeabilità della GFB alla perdita proteica, che, in ultima analisi, porta a un maggior danno alla GFB stessa (30). Da questo esempio è facile intuire come il costante sviluppo di fluorofori con sempre maggiore diversificazione, unitamente all'avanzamento nelle tecniche di transgenesi, sia in grado di ampliare fortemente le possibilità di differenti indagini di natura biologica. Ciò lo si può facilmente notare se si comparano studi sulle dinamiche dei flussi di $\mathrm{Ca}^{2+}$ tra rene e sistema nervoso centrale, in cui, per la necessità di studiare processi con velocità nettamente più elevata, i ricercatori hanno avuto la necessità di creare fluorofori con emivita e intensità differenti, come il GCaMP6 (33). Per esempio, se si volessero osservare le dinamiche che regolano la funzionalità del $\mathrm{Ca}^{2+}$ sull'intero encefalo, sarebbe impossibile farlo con il GCaMP3, al quale si è fatto riferimento relativamente al rene, in quanto ha un'emivita che si aggira sulle decine di secondi, che, nel sistema nervoso centrale, corrispondono a più ondate di $\mathrm{Ca}^{+}$, le quali sarebbero, quindi, lette sovrapposte.

Si può, quindi, intuire che, diminuendo i tempi di emissione del fluoroforo, è necessario ricorrere a tecniche di imaging capaci di acquisire il segnale di fluorescenza con tempistiche estremamente ridotte rispetto a quelle ottenibili con la microscopia confocale. Quest'ultima, infatti, essendo limitata dalla scansione punto per punto, non è in grado di campionare in modo sufficientemente veloce per seguire il segnale del $\mathrm{Ca}^{2+}$ di un potenziale di azione neuronale. Infatti, prendendo, per esempio, il cervello di larva di Zebrafish, grazie all'impiego del marcatore fluorescente GCaMP6, è stato possibile ottenere una mappatura completa dell'attività funzionale dell'intero encefalo utilizzando la microscopia a foglio di luce (34). Questa tecnica, infatti, illuminando il campione in maniera ortogonale rispetto al piano di rivelazione e acquisendo a campo largo, permette di aumentare sensibilmente la velocità di acquisizione.

Un altro ottimo esempio di come non solo la scelta di differenti fluorofori e tecniche di imaging, ma anche e soprattutto la selezione di un determinato sistema transgenico piuttosto di un altro siano in grado di generare dati e informazioni diffe- 
renti lo si ha nello studio della rigenerazione renale. È, infatti, interessante notare che, nell'ultima decade, un ampio numero di modelli transgenici è stato sviluppato con il fine di indagare sulle capacità e sulle dinamiche rigenerative del rene, di cui ancora poco è noto $(2,35)$. Per entrare più nello specifico, è possibile prendere come riferimento l'attuale dibattito sulla rigenerazione podocitaria: può questa cellula altamente specializzata e differenziata che compone la GFB dividersi e sostituire altri podociti che siano stati persi? O, altresì, la formazione de novo di podociti che si osserva in alcuni casi di malattia renale cronica $(36,37)$ è dovuta a una popolazione staminale che agisce da riserva podocitaria?

Per tentare di rispondere a questa domanda, nel tempo, sono stati messi a punto diversi sistemi transgenici basati su differenti strategie, molti dei quali, però, hanno dato luogo a opinioni contrastanti. Ciò che appare, tuttavia, sempre più chiaro è che, a causa della sua estrema specializzazione, il podocita è incapace di duplicarsi: la divisione cellulare prevederebbe infatti una variazione morfologica della cellula, la quale, per suddividere equamente i differenti componenti intracellulari e i suoi cromosomi, è necessario assuma una forma sferica. Così facendo, però, il podocita andrebbe incontro alla recessione e alla perdita dei processi pedicellari, evento in grado di indurre il denudamento della membrana basale glomerulare, con conseguente perdita della capacità di filtrazione selettiva del nefrone (38). Quindi, se la rigenerazione podocitaria avviene, essa deve essere sostenuta da un differente tipo cellulare: i podociti non possono sostituire altri podociti.

Nonostante nell'uomo sia stata dimostrata l'esistenza della popolazione di progenitori staminali parietali, o PEC, e sia stato provato che questo pool cellulare è in grado di differenziare a podocita e di migliorare la funzionalità renale quando trapiantato in modelli murini di Glomerulosclerosi Segmentale e Focale (FSGS) $(22,39)$, fino a pochi anni fa non era mai stata fornita una prova diretta del fatto che tali cellule esistessero anche nei roditori e che fossero realmente capaci di attivarsi in seguito al danno podocitario.

II primo tentativo di individuare la controparte murina delle PEC è stato effettuato nel 2009 da Appel et al. (40). Gli Autori, utilizzando un promotore inducibile costituito da un gene di fusione tra la podocalixina umana e quella di coniglio, sono stati capaci di marcare le PEC murine e di dimostrare che, nella sola fase di sviluppo renale, tali cellule sono capaci di migrare verso il tuft glomerulare acquisendo un fenotipo podocitario e agendo, pertanto, da precursori podocitari (40). Tuttavia, come evidenziato nella prima parte di questo articolo, l'uso di promotori cellulo-specifici è fondamentale per la corretta e selettiva individuazione di una popolazione di interesse, così da non cadere in errori interpretativi o dar luogo ad artefatti $(1,2,41)$. Infatti, nonostante l'interessante approccio usato da Appel et al. (40), è, comunque, da considerare che la strategia impiegata si è basata sull'uso di un promotore derivante dalla fusione di due elementi esogeni, uno umano e uno di coniglio, inseriti, però, nel genoma di topo. Non essendo fisiologicamente presente nel patrimonio genico dell'animale, il transgene costituito da questi due elementi potrebbe portare a una non corretta identificazione della popolazione di progenitori di interesse in quanto non naturalmente espresso dalle cellule di topo, così come potrebbe indurre modifiche di comportamento o fenotipiche come conseguenza della sua attivazione $(2,41)$. Tuttavia, il maggiore limite evidenziato dallo studio di Appel et al. (40) è che, fino a poco tempo fa, non si conoscevano marcatori noti e specifici che permettessero di individuare selettivamente la popolazione di PEC dei roditori, motivo per il quale gli Autori erano ricorsi all'impiego di elementi genetici esogeni che non permettono, però, di studiare la fisiologia delle PEC in modelli animali adulti. Così facendo, non solo non è possibile comprendere i meccanismi biologici che stanno alla base della rigenerazione podocitaria dell'adulto ma, soprattutto, non è possibile avere dei modelli in cui effettuare drug testing con la finalità di individuare composti in grado di attivare o di incrementare la capacità rigenerativa delle PEC. Questi studi di modulazione possono essere condotti sia in vitro, in cui, però, è persa la capacità di valutare l'effetto del farmaco nel contesto dell'organo o dell'intero organismo, che in modelli sperimentali animali in vivo, i quali, garantendo la possibilità di effettuare analisi in un contesto completamente fisiologico e sull'intero organismo, forniscono risultati molto più dettagliati e permettono una valutazione globale, oltre che specifica dell'organo d'interesse, dell'effetto della molecola (42-44).

La soluzione a questo problema è rappresentata dall'impiego di sistemi transgenici aventi come promotore un marcatore fisiologicamente e selettivamente espresso dalla popolazione che si intende studiare. Infatti, così facendo, non solo non sono introdotti elementi genomici appartenenti ad altre specie che potrebbero alterare l'interpretazione dei dati ottenuti, ma si è anche sicuri che l'attivazione del sistema transgenico avvenga, per esempio, con i medesimi tempi d'attivazione che il promotore che si è scelto avrebbe durante lo sviluppo dell'animale. Relativamente al rene, una tattica di questo genere è stata impiegata da Barker et al., i quali hanno impiegato il gene per il recettore di membrana Lgr5 (Leucinerich repeat-containing G-protein coupled receptor 5), al fine di individuare possibili popolazioni staminali renali (45). Lgr5, infatti, è già stato ampiamente caratterizzato per la sua associazione con fenotipi staminali in diversi organi di uomo e di topo, come nel caso dell'epitelio intestinale, dello stomaco, del fegato, del follicolo pilifero o del sistema ematopoietico in fase di sviluppo (46-50). Grazie a un modello murino transgenico attivato dal promotore Lgr5, Barker et al. hanno dimostrato che, nel rene fetale, esiste una popolazione staminale che guida la formazione di quello che sarà il futuro nefrone. Infatti, analisi di lineage tracing nel topo hanno evidenziato che il pool staminale Lgr5', che appare all'interno del corpo a forma di $\mathrm{S}$ intorno al quattordicesimo giorno di gestazione e che è definitivamente silenziato al giorno post-natale 7 , è 
coinvolto nella formazione delle strutture che daranno luogo al tratto spesso ascendente dell'ansa di Henle e del tubulo convoluto distale del rene adulto (45). Tuttavia, questa strategia non ha permesso di individuare popolazioni di progenitori staminali adulti, ma, bensì, solo una popolazione cellulare la cui esistenza transiente è limitata alla fase di sviluppo del nefrone: ciò preclude, pertanto, la possibilità di individuare molecole ad azione farmacologica che permettano di incrementare le potenzialità rigenerative del rene adulto.

Recentemente, applicando la stessa strategia, Lasagni et al. sono stati in grado di individuare in maniera univoca una popolazione di progenitori adulti situati nella capsula di Bowman murina. Gli autori, infatti, impiegando un modello murino guidato dal promotore Pax2 (Paired box gene 2), hanno dimostrato che l'epitelio parietale della capsula di Bowman di topo è caratterizzato dalla presenza di una popolazione di progenitori capaci di sostituire i podociti persi in seguito ad induzione di deplezione podocitaria acuta (23). Analogamente a quanto fatto da Barker et al., il promotore attivante il sistema transgenico è stato scelto in quanto Pax2 è noto per essere profondamente coinvolto nell'organogenesi del rene e perché associato a fenotipi staminali, ma, a differenza di Lgr5, la sua espressione è mantenuta anche nel rene adulto (51-54). Tuttavia, oltre ad aver provato che esiste la controparte murina delle PEC, Lasagni et al. hanno evidenziato come tali progenitori murini $\mathrm{Pax} 2^{+}$siano in grado di attivarsi solo qualora il danno podocitario sia di lieve entità e si abbia, pertanto, una limitata perdita proteica (23). Al contrario, qualora il danno sia di maggior rilievo, l'albumina persa dalla GFB sequestra l'acido retinoico endogeno, il quale è, poi, perso nelle urine complessato con tale proteina: ciò impedisce ai progenitori renali di attivarsi mettendo in atto le loro attività pro-rigenerative $(23,55)$. Per questo motivo, è spesso possibile osservare, in ambito clinico, pazienti che vanno incontro a una spontanea remissione della proteinuria qualora questa sia limitata, mentre, se il danno è di entità medio-forte, allora il decorso proteinurico sarà incrementale e il paziente svilupperà una maggiore glomerulosclerosi.

Il punto di maggior rilievo di questo studio è che, essendo stati identificati per la prima volta i progenitori renali endogeni di topo adulto, Lasagni et al. hanno potuto testare vari composti che potessero modulare il danno renale e indurre rigenerazione anche in quegli animali con forte proteinuria. Tra le molecole valutate, in particolare, un inibitore dell'enzima GSK3 (Glycogen synthase kinase 3) si è dimostrato capace di incrementare notevolmente la remissione dalla proteinuria, in quanto capace di sensibilizzare i progenitori renali all'effetto dell'acido retinoico endogeno (23). Per primo, quindi, tale studio ha proposto una strategia terapeutica farmacologica volta a modulare l'attività dei progenitori endogeni così da aumentarne le capacità rigenerative, rimarcando, quindi, l'importanza dei modelli transgenici per lo studio della fisiopatologia e della rigenerazione renale. Allo stesso modo, questo studio ci permette anche di valutare il grande impatto che hanno per la ricerca le più recenti tecniche di imaging: I'uso della MPM e la marcatura nucleare con DAPI, infatti, hanno permesso a Lasagni et al. di contare finemente il numero di cellule $\mathrm{Pax}^{+}$che, migrando sul tuft glomerulare, hanno acquisito morfologia e marcatori tipicamente associati al podocita, permettendo, inoltre, di dimostrare che l'inibizione della GSK3 è in grado di promuovere la loro attività rigenerativa (23).

Tutti gli esempi citati finora ci hanno permesso di analizzare come diverse soluzioni di transgenesi e metodiche di imaging possano essere utilizzate per lo studio di fini processi biologici e come, a seconda delle differenti strategie scelte, si possano ricavare certe informazioni piuttosto che altre. Malgrado ciò, va ricordato che la ricerca di natura preclinica si pone come obiettivo quello di implementare le nostre conoscenze di base, così che queste possano avere una ricaduta di natura clinica e garantiscano un miglior approccio terapeutico nella cura del paziente. Per questo motivo è interessante analizzare come le tecniche descritte contribuiscano a migliorare la pratica clinica e come alcune di queste siano tutt'oggi direttamente impiegabili in diagnostica.

\section{"Imaging traslazionale": applicazioni clinico-diagnos- tiche delle tecnologie sviluppate in ricerca}

Anche se di estremo rilievo nel campo della ricerca e per quanto ci chiarifichino con estrema precisione i meccanismi fisiopatologici che regolano omeostasi, rigenerazione e differenti condizioni patologiche rivelandone le dinamiche nel contesto d'organo, le innovazioni legate alla transgenesi non possono che rimanere confinate all'ambito sperimentale e preclinico. Tuttavia, se le tecniche di transgenesi si fermano a tal punto e servono prevalentemente a orientare le nostre conoscenze così da indirizzarci verso il corretto approccio clinico, le tecniche di imaging analizzate finora possono essere facilmente impiegate anche per la valutazione diagnostica in ambito clinico. Ciò non solo ci rende conto dell'importanza dei modelli sperimentali su cui sono studiati i principi biologici dei meccanismi che regolano la vita di un organismo, ma, allo stesso tempo, spiega perché, soprattutto nel campo dell'imaging, siano costantemente introdotte nuove metodiche e strumentazioni con cui sia possibile valutare differenti tessuti di pazienti affetti da diverse patologie.

La prima osservazione biologica di singole cellule risale al 1655 e fu effettuata da Robert Hooke che, successivamente, fu in grado di migliorare le qualità dell'osservazione microscopica a tal punto da essere definito il padre della microscopia moderna. Quest'ultimo inventò il primo microscopio ottico "standard" o, semplicemente, microscopio ottico, composto da un sistema di lenti e specchi che ingrandisce un'immagine e raccoglie a campo largo la luce con un obiettivo posto sul campione. Oggi le tecniche di microscopia ottica sono più avanzate e impiegano, generalmente, la fluorescenza (microscopia confocale e MPM), motivo per cui, quando applicate 
alla clinica, permettono di ottenere immagini di qualità nettamente superiori rispetto alla microscopia ottica "standard". Tuttavia, nonostante quest'ultima resti la strumentazione ottica senza dubbio più impiegata per tempistica, facilità di utilizzo e costi relativamente contenuti, presenta comunque dei limiti intriseci che la rendono limitata a uno studio più grossolano.

Da un punto di vista cronologico, nel campo della nefrologia, le prime tecniche che hanno permesso di effettuare analisi su campioni espiantati da pazienti sono state quelle basate sull'uso di coloranti, ossia sostanze chimico-biologiche che, legandosi a determinate componenti cellulari, ne aumentano il contrasto permettendo, così, all'operatore di osservare le strutture di interesse $(56,57)$. Nonostante le tecniche colorimetriche permettano analisi principalmente a livello macroscopico, molte di queste sono ancora comunemente usate sia in ambito clinico che in ricerca, come nel caso dell'ematossilina-eosina (58-60). Questa è sicuramente la colorazione più nota e impiegata, in quanto consente lo studio topografico del tessuto, che apparirà con i nuclei cellulari marcati in blu e il citoplasma in rosa. Ciò garantisce non solo di distinguere le principali strutture che formano il nefrone, ma permette anche di documentare la dilatazione tubulare, l'accumulo parenchimale di cellule del sistema immunitario e la formazione di depositi intra-tubulari tipicamente associati a insufficienza renale acuta (AKI). In questo caso è possibile: valutare se il tessuto bioptico di un paziente presenti o meno segni di AKI e di che intensità, a quando approssimativamente si deve far risalire l'evento che ha causato I'AKI e quale sia l'estensione dell'area danneggiata. Molto impiegata per tale scopo è la colorazione denominata Tricromica di Masson, che, grazie all'impiego di tre diversi coloranti, permette di individuare $i$ nuclei in viola, il citoplasma in rosso tenue e in blu il tessuto connettivo, indice, quest'ultimo, del grado di fibrosi di un preparato tissutale $(61,62)$. Pertanto, la Tricromica di Masson è prevalentemente utilizzata per valutare il grado di danneggiamento del tessuto renale e per creare degli scoring di danno, anche se tali gradazioni risentono della bassa capacità risolutiva della microscopia ottica "standard" e della variabilità operatore-dipendente.

I tessuti su cui si intende fare uso di coloranti o marcature basate su anticorpi devono essere protetti da alterazioni autolitiche e, pertanto, devono essere fissati nel più breve tempo possibile tramite quelli che sono chiamati fissativi (63). La fissazione non serve solo a immobilizzare i differenti costituenti cellulari del campione in uno stato quanto più possibile simile a quello che hanno fisiologicamente, ma garantisce, inoltre, al preparato di sopportare stress chimici e fisici come variazioni di temperatura, sezionamenti o reazioni chimico-enzimatiche finalizzate alla marcatura del tessuto. Inoltre, affinché la fissazione avvenga in maniera ottimale e omogenea su tutto il tessuto, generalmente si consiglia che i frammenti di tessuto non superino il $\mathrm{cm}$ di diametro, come nel caso delle biopsie renali espiantate a fini diagnostici. Tut- tavia, le procedure di fissazione possono alterare la struttura di determinati antigeni e, quindi, la loro capacità di essere legati da anticorpi, o possono bloccare o modificare sostanzialmente la capacità di far avvenire alcune reazioni enzimatiche sul tessuto in esame. Per tale motivo, essendo i protocolli di fissazione tra i più vari e differenti, questi sono scelti in base al tipo di analisi, di colorazione e processamento che si intende effettuare sul preparato. Per esempio, le fissazioni che mascherano le componenti proteiche cellulari possono difficilmente essere impiegate qualora si abbia intenzione di effettuare analisi immunomediate o basate su reazioni enzimatiche.

Successivamente alla considerazione che la maggior parte delle molecole biologiche sono antigeni che possono essere riconosciuti e legati da anticorpi e grazie all'incremento delle conoscenze nel campo immunologico, il grosso della diagnostica per imaging si è spostata su marcature immunologiche $(64,65)$. Tra queste marcature, la prima sviluppata e a essersi diffusa è stata l'immunoistochimica, la quale sfrutta anticorpi previamente coniugati con l'enzima perossidasi al fine di rivelare specifici antigeni e strutture cellulari. Infatti, ai tessuti incubati con l'anticorpo coniugato all'enzima, è successivamente aggiunto un substrato che la perossidasi trasforma in un prodotto colorato nel punto esatto di legame dell'anticorpo, rivelando, perciò, la localizzazione dell'antigene. Tuttavia, anche se di largo utilizzo, le tecniche di immunoistochimica sono sempre più rimpiazzate da tecniche di immunofluorescenza, le quali prevedono l'utilizzo di anticorpi coniugati a fluorofori. Ciò è principalmente dovuto al fatto che, mentre per l'immunoistochimica il principale mezzo di rilevazione è il microscopio ottico "standard", al contrario l'immunofluorescenza necessita della microscopia ottica a fluorescenza, della microscopia confocale o della microscopia a multifotoni, tutti strumenti con un potere risolutivo decisamente maggiore. II concetto su cui si basa l'immunofluorescenza è lo stesso dell'immunoistochimica: si trattano i campioni biologici di cui si vuole mettere in evidenza un determinato antigene con un anticorpo, il quale è, in questo caso, coniugato a una molecola capace di emettere in fluorescenza quando eccitata a precise $\lambda$. Entrambi i tipi di saggi immunomediati hanno il grande vantaggio di offrire un'elevata specificità: gli anticorpi possono, infatti, essere diretti virtualmente verso ogni tipo di molecola biologica, come determinate isoforme enzimatiche, strutture intracellulari con alterazioni note, proteine di membrana e via dicendo. Questo livello di specificità, comunque sia inferiore rispetto a quello ottenibile mediante approcci di natura genetica riconducibili all'uso di modelli transgenici, è più che sufficiente per le analisi condotte routinariamente in clinica.

Un ottimo esempio è la glomerulonefrite a depositi mesangiali di IgA: per diagnosticare la patologia si va a incubare il campione bioptico del paziente con anticorpi coniugati a fluorofori e diretti contro le IgA umane. Se, nel tessuto del paziente, dovessero essere presenti depositi di IgA a livello glomerulare, l'anticorpo anti-IgA vi si legherà con alta affinità 
e sarà, poi, rilevabile in fluorescenza, preferibilmente in microscopia confocale o multifotoni. Microscopio confocale e MPM permettono, quindi, di effettuare una diagnosi molto accurata non solo di natura qualitativa, ma anche quantitativa: è, infatti, possibile effettuare uno score ottico del grado di estensione e della quantità di depositi, effettuato generalmente da più operatori indipendenti. Tuttavia, la microscopia a fluorescenza in diagnostica non si avvale solo dell'uso di anticorpi fluorescenti ma, per esempio, grazie ai coloranti nucleari HOECHST 33342 o DAPI permette di valutare la morfologia nucleare o di contare i nuclei cellulari per verificare la presenza di deplezioni podocitarie acute o l'apparizione di crescent nel caso di una glomerulonefrite rapidamente progressiva. Da questo si capisce l'estrema importanza dell'utilizzo delle tecniche di microscopia a fluorescenza in clinica che, garantendo un'accuratezza di alcune centinaia di nm con un'invasività minore rispetto a quella della microscopia elettronica, permettono di avere immagini più complete da un punto di vista strutturale e più specifiche per l'individuazione di una determinata malattia. Inoltre, grazie agli ultimi sviluppi delle tecniche di schiarimento e di colorazione, oggi come oggi si riescono ad analizzare campioni molto più spessi e con contrasti migliori, ottenendo immagini di qualità elevata delle specifiche strutture legate dall'anticorpo collegato al fluoroforo.

Come già introdotto precedentemente, i vantaggi della microscopia ottica "standard" per le applicazioni in clinica sono le grandi affidabilità, semplicità e vantaggiosità commerciale rispetto alle altre tecniche di imaging. Basti pensare che, per effettuare analisi con un microscopio ottico bastano una struttura posizionabile su un tavolo e un computer che si interfacci con l'operatore grazie a sistemi operativi di acquisizione. Questi, negli ultimi anni, sono stati oggetto di un forte avanzamento tecnologico, diventando, allo stesso tempo, sempre più intuitivi e approcciabili anche per i non esperti $(66,67)$. Inoltre, la microscopia ottica "standard" garantisce un range dinamico vasto in termini di ingrandimenti e spesso non necessita di tecniche di schiarimento troppo aggressive, come nel caso della microscopia elettronica che, per rendere opache agli elettroni le strutture biologiche, utilizza metalli pesanti come oro e piombo.

Tuttavia, se si vogliono osservare dettagli ad alta risoluzione, il gold standard in clinica rimane la microscopia elettronica che, ottenendo immagini del tessuto dall'attraversamento di un fascio di elettroni fortemente accelerato, non necessita della presenza di molecole fluorescenti per l'acquisizione di immagini di fini componenti ultrastrutturali e processi biologici. Infatti, gli elettroni, attraversando una sezione ultrasottile $(50-70 \mathrm{~nm})$ del campione, permettono una visualizzazione del preparato in scala di grigi con un'estrema risoluzione e con ingrandimenti che arrivano sino a 3-4 nm (4). Per queste caratteristiche, la microscopia elettronica in ambito clinico nefrologico è prevalentemente usata per valutare alterazioni ultrastrutturali delle cellule, come l'appiattimento delle cellule dell'epitelio tubulare e la loro perdita dell'orletto a spaz- zola, tipicamente associati a episodi di AKI, o per quantificare retrazione e perdita dei processi pedicellari podocitari in caso di malattia renale cronica. Tuttavia, il limite della microscopia a elettroni è che si può perdere la "visione generale" dell'intera struttura o del tessuto che si vogliono studiare, in quanto i frammenti sezionati per tale tipo di microscopia non possono generalmente superare $1 \mathrm{~mm}$ di diametro. Va, però, considerato che questa è una tecnica non routinaria, di difficile esecuzione e temporalmente ed economicamente dispendiosa, motivo per cui, generalmente, vi si ricorre solo in caso di patologia già conclamata, di cui si vogliano, però, capire e studiare alcuni aspetti che potrebbero essere clinicamente determinanti nella scelta di una terapia piuttosto di un'altra.

Uno studio di metanalisi ha dimostrato, infatti, che la microscopia elettronica ha permesso di correggere diagnosi già effettuate nell' $11 \%$ dei casi presi in analisi, che è servita a confermare la diagnosi nel $21 \%$ dei casi che hanno fatto ricorso a tale tecnica di imaging e che, addirittura nel 3\% della casistica, è stato possibile effettuare una differente diagnosi del tutto non correlata a quella iniziale (68).

Da tutto ciò deduciamo che, in ambito medico, sono impiegate le più disparate tecniche di imaging: dalla MRI, passando per la microscopia ottica, fino ad arrivare alla microscopia elettronica, permettendo, così, di spaziare in un ampio range di ingrandimenti e risoluzioni che garantiscono un'analisi più dettagliata delle patologie umane.

\section{Conclusioni}

Quando elaborati modelli animali transgenici sono combinati con le più avanzate tecniche di imaging, si ottengono strumenti estremamente potenti che permettono ai ricercatori di comprendere i meccanismi fisiopatologici e le dinamiche cellulari del rene così come di altri organi. Ciò che ha maggior rilevanza, però, è il fatto che le conoscenze derivanti dallo studio via imaging in modelli animali geneticamente modificati possono essere traslate in ambito clinico, con importanti ricadute che permettono di studiare tessuti patologici e consentono di eseguire diagnosi con estrema rapidità e, soprattutto, affidabilità e precisione. Tuttavia, le limitazioni intrinseche di transgenesi e imaging devono essere accuratamente valutate nella scelta della corretta progettazione sperimentale, così come delle tecniche diagnostiche che si vogliono usare, affinché l'analisi dei risultati e l'interpretazione dei dati siano corrette e accurate. Malgrado ciò, le innovazioni in entrambi i campi non solo stanno cambiando la nostra visione sulla capacità rigenerativa del rene, ma stanno anche rendendo accessibile alla nostra conoscenza una vasta gamma di processi biologici che possono, ora, essere direttamente visualizzati sia in tessuti espiantati che in modelli animali in vivo nel momento in cui avvengono. Ottimo esempio di come l'applicazione combinata di transgenesi e imaging abbia aperto nuove strade nella comprensione della fisiologia renale è stato la strada che ha portato all'individuazione, nel 
topo, di un pool di progenitori di natura staminale che funge da riserva podocitaria e che può essere modulato farmacologicamente, così da incrementarne le capacità rigenerative. È, pertanto, facile intuire come tutte le tecnologie a cui si è fatto riferimento in questo contributo e che sono in costante sviluppo possano aprire ogni giorno nuove strade che ci consentono di avere una più profonda comprensione di funzioni e dinamiche cellulari d'organo, le quali possono, poi, essere traslate per l'applicazione clinica, garantendo, di conseguenza, una miglior affidabilità in diagnosi e cura.

\section{Disclosures}

Financial support: No financial support was received for this submission.

Conflict of interest: The authors have no conflict of interest.

\section{Bibliografia}

1. Lombardi D, Alterini T. New techniques of transgenesis and imaging: applications in Neurology and Nephrology. Part I. Giornale di Tecniche Nefrologiche e Dialitiche 2014;26(2):173-81.

2. Lombardi D, Lasagni L. Transgenic Strategies to Study Podocyte Loss and Regeneration. Stem Cells Int 2015;2015: 678347.

3. Alexander AL. Diffusion tensor imaging of the brain. Neurotherapeutics 2007;4(3):316-29.

4. Denk W, Horstmann H. Serial block-face scanning electron microscopy to reconstruct three-dimensional tissue nanostructure. PLoS Biol 2004;2(11):e329.

5. Starborg T. Using transmission electron microscopy and 3View to determine collagen fibril size and three-dimensional organization. Nat Protoc 2013;8(7):1433-48.

6. Oheim M. Two-photon microscopy in brain tissue: parameters influencing the imaging depth. J Neurosci Methods 2001;111 (1):29-37.

7. Lichtman JW, Conchello JA. Fluorescence microscopy. Nat Methods 2005;2(12):910-9.

8. Kasthuri N, Lichtman JW. The rise of the 'projectome'. Nat Methods 2007;4(4):307-8.

9. Mertz J. Optical sectioning microscopy with planar or structured illumination. Nat Methods 2011;8(10):811-9.

10. Grgic I. Imaging of podocyte foot processes by fluorescence microscopy. J Am Soc Nephrol 2012;23(5):785-91.

11. Hell SW, Wichmann J. Breaking the diffraction resolution limit by stimulated emission: stimulated-emission-depletion fluorescence microscopy. Opt Lett 1994;19(11):780-2.

12. Henriques R, Mhlanga MM. PALM and STORM: what hides beyond the Rayleigh limit? Biotechnol J 2009;4(6):846-57.

13. Kim AH, Suleiman $H$, Shaw AS. New approaches in renal microscopy: volumetric imaging and superresolution microscopy. Curr Opin Nephrol Hypertens 2016;25(3):159-67.

14. Conchello JA, Lichtman JW. Optical sectioning microscopy. Nat Methods 2005;2(12):920-31.

15. Patterson GH, Piston DW. Photobleaching in two-photon excitation microscopy. Biophys J 2000;78(4):2159-62.

16. Zipfel WR, Williams RM, Webb WW. Nonlinear magic: multiphoton microscopy in the biosciences. Nat Biotechnol 2003;21(11):1369-77.

17. Helmchen F, Denk W. Deep tissue two-photon microscopy. Nat Methods 2005;2(12):932-40.

18. Huisken J, Stainier DY. Selective plane illumination microscopy techniques in developmental biology. Development 2009;136 (12):1963-75.

19. Hackl MJ. Tracking the fate of glomerular epithelial cells in vivo using serial multiphoton imaging in new mouse models with fluorescent lineage tags. Nat Med 2013;19(12):1661-6.

20. Burger D. Urinary podocyte microparticles identify prealbuminuric diabetic glomerular injury. J Am Soc Nephrol 2014;25(7):1401-7.

21. Wickman L. Urine podocyte mRNAs, proteinuria, and progression in human glomerular diseases. J Am Soc Nephrol 2013;24(12):2081-95.

22. Sagrinati $C$. Isolation and characterization of multipotent progenitor cells from the Bowman's capsule of adult human kidneys. J Am Soc Nephrol 2006;17(9):2443-56.

23. Lasagni L. Podocyte Regeneration Driven by Renal Progenitors Determines Glomerular Disease Remission and Can Be Pharmacologically Enhanced. Stem Cell Reports 2015;5(2):248-63.

24. Rankin EB, Tomaszewski JE, Haase VH. Renal cyst development in mice with conditional inactivation of the von Hippel-Lindau tumor suppressor. Cancer Res 2006;66(5):2576-83.

25. Lasagni L, Romagnani P. Glomerular epithelial stem cells: the good, the bad, and the ugly. J Am Soc Nephrol 2010;21(10):1612-9.

26. SchiessI IM, Bardehle $S$, Castrop $H$. Superficial nephrons in $\mathrm{BALB} / \mathrm{C}$ and $\mathrm{C} 57 \mathrm{BL} / 6$ mice facilitate in vivo multiphoton microscopy of the kidney. PLoS One 2013;8(1):e52499.

27. Devi S. Multiphoton imaging reveals a new leukocyte recruitment paradigm in the glomerulus. Nat Med 2013;19(1): 07-12.

28. Peti-Peterdi J, Multiphoton imaging of renal regulatory mechanisms. Physiology (Bethesda) 2009;24:88-96.

29. Peti-Peterdi J, Sipos A. A high-powered view of the filtration barrier. J Am Soc Nephrol 2010;21(11):1835-41.

30. Burford JL. Intravital imaging of podocyte calcium in glomerular injury and disease. J Clin Invest 2014;124(5):2050-8.

31. Salmon AH. Loss of the endothelial glycocalyx links albuminuria and vascular dysfunction. J Am Soc Nephrol 2012;23(8): 1339-50.

32. Nakano D. Multiphoton imaging of the glomerular permeability of angiotensinogen. J Am Soc Nephrol 2012;23(11):1847-56.

33. Chen TW. Ultrasensitive fluorescent proteins for imaging neuronal activity. Nature 2013;499(7458):295-300.

34. Ahrens MB. Whole-brain functional imaging at cellular resolution using light-sheet microscopy. Nat Methods 2013;10 (5):413-20.

35. Lombardi D, Becherucci F, Romagnani P. How much can the tubule regenerate and who does it? An open question. Nephrol Dial Transplant 2015.

36. Pavenstadt $\mathrm{H}$, Kriz W, Kretzler M. Cell biology of the glomerular podocyte. Physiol Rev 2003;83(1):253-307.

37. Remuzzi G, Benigni A, Remuzzi A. Mechanisms of progression and regression of renal lesions of chronic nephropathies and diabetes. J Clin Invest 2006;116(2):288-96.

38. Lasagni L. Notch activation differentially regulates renal progenitors proliferation and differentiation toward the podocyte lineage in glomerular disorders. Stem Cells 2010;28(9):1674-85.

39. Ronconi E. Regeneration of glomerular podocytes by human renal progenitors. J Am Soc Nephrol 2009;20(2):322-32.

40. Appel D. Recruitment of podocytes from glomerular parietal epithelial cells. J Am Soc Nephrol 2009;20(2):333-43.

41. Romagnani P, Rinkevich $Y$, Dekel B. The use of lineage tracing to study kidney injury and regeneration. Nat Rev Nephrol 2015; 11(7):420-31.

42. Schaun MI. Cell Therapy in Ischemic Heart Disease: Interventions That Modulate Cardiac Regeneration. Stem Cells Int 2016; 2016: 2171035.

43. Smeriglio P. Phosphotyrosine phosphatase inhibitor bis- 
peroxovanadium endows myogenic cells with enhanced muscle stem cell functions via epigenetic modulation of Sca-1 and Pw1 promoters. FASEB J 2015.

44. Shi J, Longo FM, Massa SM. A small molecule p75(NTR) ligand protects neurogenesis after traumatic brain injury. Stem Cells 2013;31(11):2561-74.

45. Barker N. Lgr5(+ve) stem/progenitor cells contribute to nephron formation during kidney development. Cell Rep 2012;2(3): 540-52.

46. Barker N. Identification of stem cells in small intestine and colon by marker gene Lgr5. Nature 2007;449(7165):1003-7.

47. Barker N. Lgr5(+ve) stem cells drive self-renewal in the stomach and build long-lived gastric units in vitro. Cell Stem Cell 2010;6(1):25-36.

48. Huch M. In vitro expansion of single Lgr5+liver stem cells induced by Wnt-driven regeneration. Nature 2013.494(7436):247-50.

49. Jaks V. Lgr5 marks cycling, yet long-lived, hair follicle stem cells. Nat Genet 2008;40(11):1291-9.

50. Liu D. Leucine-rich repeat-containing G-protein-coupled Receptor 5 marks short-term hematopoietic stem and progenitor cells during mouse embryonic development. J Biol Chem 2014;289(34):23809-16.

51. Dressler GR. Epigenetics, development, and the kidney. J Am Soc Nephrol 2008;19(11):2060-7.

52. Dressler GR. The specification and maintenance of renal cell types by epigenetic factors. Organogenesis 2009;5(2):73-82.

53. Sallustio F. TLR2 plays a role in the activation of human resident renal stem/progenitor cells. FASEB J 2010;24(2):514-25.

54. Loverre A. Increase of proliferating renal progenitor cells in acute tubular necrosis underlying delayed graft function. Transplantation 2008;85(8):1112-9.

55. Peired A. Proteinuria impairs podocyte regeneration by sequestering retinoic acid. J Am Soc Nephrol 2013;24(11): 1756-68.
56. Wachstein M. Histochemical staining reactions of the normally functioning and abnormal kidney. J Histochem Cytochem 1955;3(4):246-70.

57. Cooper WG, Reger JF, Van Breemen VL. Observations on the basement membranes in rat kidney. J Biophys Biochem Cyto 1956;2(4 Suppl.):283-6.

58. Penney DP. Analysis and testing of biological stains--the Biological Stain Commission Procedures. Biotech Histochem 2002;77(5-6):237-75.

59. Bonsib SM, Reznicek MJ. Renal biopsy frozen section: a fluorescent study of hematoxylin and eosin-stained sections. Mod Pathol 1990;3(2):204-10.

60. Sims-Lucas S. Analysis of 3D branching pattern: hematoxylin and eosin method. Methods Mol Biol 2012;886:73-86.

61. Cohen AH. Masson's trichrome stain in the evaluation of renal biopsies. An appraisal. Am J Clin Pathol 1976;65(5):631-43.

62. Farris $A B$. Morphometric and visual evaluation of fibrosis in renal biopsies. J Am Soc Nephrol 2011;22(1):176-86.

63. Hopwood D. Fixatives and fixation: a review. Histochem J 1969;1(4):323-60.

64. Molne J, Breimer ME, Svalander CT. Immunoperoxidase versus immunofluorescence in the assessment of human renal biopsies. Am J Kidney Dis 2005;45(4):674-83.

65. Schena FP, Gesualdo L. Renal biopsy--beyond histology and immunofluorescence. Nephrol Dial Transplant 1994;9(11): 1541-4.

66. Pepperkok R, Ellenberg J. High-throughput fluorescence microscopy for systems biology. Nat Rev Mol Cell Biol 2006;7(9):690-6.

67. Leblond F. Pre-clinical whole-body fluorescence imaging: Review of instruments, methods and applications. J Photochem Photobiol B 2010;98(1):77-94.

68. Haas M. A reevaluation of routine electron microscopy in the examination of native renal biopsies. J Am Soc Nephrol $1997 ; 8(1): 70-6$. 